\title{
A NEW APPROACH FOR IDENTIFYING SLEEP APNEA SYNDROME USING WAVELET TRANSFORM AND NEURAL NETWORKS
}

\author{
Robert Lin ${ }^{1}$, Ren-Guey LeE ${ }^{2}$, Chwan-Lu Tseng ${ }^{3}$, Heng-Kuan Zhou ${ }^{4}$, Chin-Feng Chao ${ }^{1}$, \\ JOE-AIR JIANG ${ }^{1}$ \\ ${ }^{1}$ Department of Bio-Industrial Mechatronics Engineering, National Taiwan University, \\ ${ }^{2}$ Institute of Computer and Communication Engineering, National Taipei University of \\ Technology, \\ ${ }^{3}$ Department of Electrical Engineering, National Taipei University of Technology, Taipei, \\ ${ }^{4}$ Department of Electrical Engineering, Lunghwa University of Science and Technology, \\ Taoyuan, Taiwan
}

\begin{abstract}
This paper describes a new technique to classify and analyze the electroencephalogram (EEG) signal and recognize the EEG signal characteristics of Sleep Apnea Syndrome (SAS) by using wavelet transforms and an artificial neural network (ANN). The EEG signals are separated into Delta, Theta, Alpha, and Beta spectral components by using multi-resolution wavelet transforms. These spectral components are applied to the inputs of the artificial neural network. We treated the wavelet coefficient as the kind of the training input of artificial neural network, might result in 6 groups of wavelet coefficients per second signal by way of characteristic part processing technique of the artificial neural network designed by our group, we carried out the task of training and recognition of SAS symptoms. Then the neural network was configured to give three outputs to signify the SAS situation of the patient. The recognition threshold for all test signals turned out to have a sensitivity level of approximately $69.64 \%$ and a specificity value of approximately $44.44 \%$. In neurology clinics, this study offers a clinical reference value for identifying $S A S$, and could reduce diagnosis time and improve medical service efficiency.
\end{abstract}

Biomed Eng Appl Basis Comm, 2006(June); 18: 138-143.

Keywords: EEG; sleep apnea syndrome; wavelet transform.

\section{INTRODUCTION}

Received: Jan. 20, 2006; Accepted: April 6, 2006

Correspondence: Joe-Air Jiang, Professor

Department of Bio-Industrial Mechatronics

Engineering, National Taiwan University, Taipei,

Taiwan

E-mail: jajiang@ntu.edu.tw
Humans spend nearly one-third of their lives sleeping. Good sleep is an essential precondition to the maintenance of mental and physical health. As people enter middle age and beyond, the upper respiratory tracts of some individuals shrink, which may lead to obstruction of the nasal passages and snoring during sleep. This apnea may affect the quality of sleep and health when it occurs frequently, and may even cause death in severe cases. Sleep Apnea Syndrome (SAS) is a very common sleep disorder. Breathing often stops 
for more than ten seconds each instance in a case of SAS, after which the patient must struggle to return to a normal state of breathing. This continues in cyclic fashion. A patient is considered to suffer from SAS if he or she suffers breathing stoppages an average of five times per hour for more than ten seconds each time. However, after a patient awakens because of SAS, the resumption of breathing must occur continuously for more than 3 seconds [1]. SAS is usually diagnosed by analysis of clinical polysomnography (PSG) signals, which measure the chest and abdominal breathing effort, nasal and oral airflow, and blood-oxygen ( $\mathrm{SpO} 2)$ saturation, and by electrocardiogram (ECG).

Electroencephalogram (EEG) signals are quite useful since they give off no radiation, are noninvasive, and are suitable for monitoring over long periods of time. In addition, useful EEG reference values are available for studying SAS. EEG signals are neither stationary nor arbitrarily shifted in frequency range, and can be classified as four types of basis waves, namely as delta, theta, alpha, and beta waves. There is an abrupt frequency shift in the EEG signal when sleep apnea ends: Sleep EEG activity shifts from a delta wave to theta and alpha waves in the range of $4 \sim 14 \mathrm{~Hz}$. This phenomenon is used as a criterion for identifying SAS [2].

SAS may occur for 10 seconds or more when a patient falls asleep during non-rapid eye movement (NREM) sleep. When breathing becomes normal, brain waves tend to shift to a relatively continuous frequency signal above delta, namely in the theta and alpha wave frequency bands [3-4]. If we compare EEG signals with nasal and oral airflow signals when symptoms of SAS, we see that the nasal and oral airflow signals are clearly reduced, and the EEG signal shifts above the delta frequency band, when an episode of SAS occurs. As shown in Fig. 1, the EEG signal is continuous at a high frequency level. This signal is a characteristic sign of the SAS disorder.
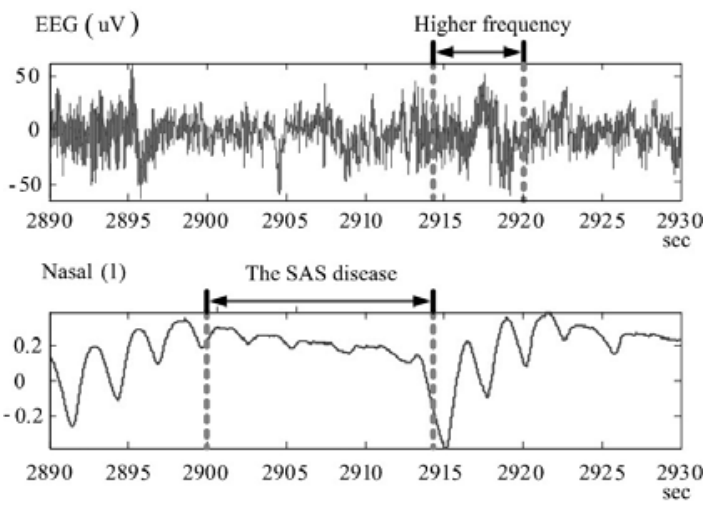

Fig. 1. The signal characteristic of SAS disease.
Records from a sleep database (the MIT-BIH Polysomnography Database) were analyzed over 30second time intervals to determine the patient's sleep condition. Taking the slp59 EEG record as an example, the signal sampling frequency was $250 \mathrm{~Hz}$, total recorded time was 4 hours, and the entire sleep time was divided into 480 small episodes; each episode was separately judged, and was recorded in the file slp59.st. Our system was used to sequentially analyze EEG records via the steps of training, simulation testing, and adjustment of the learning rate parameter, etc., and the parameter was tested and adjusted within the tolerable range of error. Artificial neural network (ANN) training was performed in this paper in order to achieve effective recognition results. The system's recognition results were compared with doctors' recognition findings in the MIT-BIH sleep database. Table I provides the definitions of sensitivity and specificity. A true positive (TP) signifies that a SAS seizure was recognized both by a doctor and by the computer system. A false negative $(\mathrm{FN})$ signifies that a SAS seizure was identified by the computer system, but not by the doctor. A false positive (FP) signifies that the SAS seizure was identified by the doctor, but not by the computer. A true negative (TN) signifies that the SAS seizure was identified by neither the doctor nor the computer system.

Table I. The definitions of sensitivity and specificity

\begin{tabular}{|c|c|c|}
\hline System & $\begin{array}{c}\text { SAS } \\
\text { ( Positive ) }\end{array}$ & $\begin{array}{c}\text { SAS } \\
\text { ( Negative ) }\end{array}$ \\
\hline $\begin{array}{c}\text { SAS } \\
\text { ( Positive ) }\end{array}$ & $\begin{array}{l}\text { True Positive } \\
\text { ( TP ) }\end{array}$ & $\begin{array}{l}\text { False Negative } \\
\text { ( FN ) }\end{array}$ \\
\hline \multirow[t]{2}{*}{$\begin{array}{c}\text { SAS } \\
(\text { Negative ) }\end{array}$} & $\begin{array}{l}\text { False Positive } \\
\text { (FP ) }\end{array}$ & $\begin{array}{l}\text { True Negative } \\
\text { (TN ) }\end{array}$ \\
\hline & $\begin{array}{c}\text { Sensitivity } \\
(\mathrm{TP} / \mathrm{TP}+\mathrm{FP})\end{array}$ & $\begin{array}{c}\text { Specificity } \\
(\mathrm{TN} / \mathrm{FN}+\mathrm{TN})\end{array}$ \\
\hline
\end{tabular}

\section{METHOD}

This paper used three different types of structural methods identify and compare the EEG signals characteristic of STATUS; these methods involved the use of wavelet transforms and an artificial neural network. Because it was easily able to perform signal decomposition, reconstruction, and time domain partial location, the db3 mother wavelet basis of the discrete wavelet transform (DWT) built into MATLAB was adopted to analyze EEG signals. In addition, the ANN supervised learning back-propagation algorithm was adopted and used in conjunction with the gradient steepest descent (GST) method to minimize the mean square error between the actual output and the expected output. We arrived at the best learning 
efficiency and good recognition results by changing or adjusting the weight value of the learning rule. This enabled us to distinguish and compare EEG signal characteristics using three types of different structural techniques in the ANN model; these techniques consisted of characteristic part analysis (CPA), continual characteristic segment analysis (CCSA), and characteristic segment part analysis (CSPA). The three different structural techniques model are described as follows:

\subsection{CPA Technique}

Since an entire section of the EEG signal characteristics band for SAS sleep is continuous over 13 seconds, we therefore had to simultaneously concatenate 12 -second signals from the previous section or the next section in order to extract EEG signal characteristics. We therefore used a sampling time smaller than 1 second to implement the section sampling. A signal was extracted for each second and wavelet transform decomposition performed for 0.5 second intervals (125 sampling points) in order to achieve better resolution. This allowed us to arrive at the wavelet coefficient corresponding to the wavelet transform decomposition, and this wavelet coefficient was within the scope of theta and alpha wave frequencies. We treated the wavelet coefficient as the training input for the four-layer ANN, where the ANN learns to transform an $n$-dimensional input vector into an $m$-dimensional output vector according to certain (not necessarily known) criteria. The ANN is composed of a set of $n$ simple neural computing elements. Functionally, there are four layers of cells: input, output, and interior or hidden cells. The input layer contains 12 processing elements and the first hidden layer contains 24 processing elements. The second hidden layer contains 12 processing elements and the output layer contains one processing element. Weight matrices $W$ specify the weights of the connections between cells in the four layers. Such matrices completely determine the connectivity of networks and the direction of signal propagation. The weights of connection layers are modulated in order to allow the tasks of training and classification to be carried out. We set a learning rate parameter of 0.01 so that the system would train 10,000 times. The ANN employed a sigmoid function to restrict the output value within a range of -1 to +1 . Nasal and oral airflow signals were considered auxiliary reference marks for identifying the occurrence of SAS episodes. A -1 output value indicates that a SAS seizure did not occur and $\mathrm{a}+1$ value indicates a SAS seizure. We input all the training materials and used computer programming to adjust the threshold value to set up a group model. This model expressed the conditions for identification of SAS seizures shown in Fig. 2 [5].

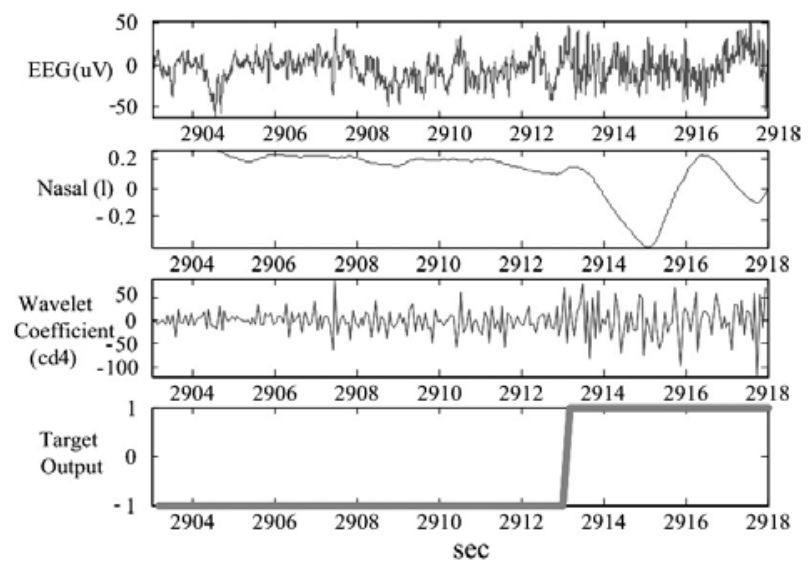

Fig. 2. The output goal value defined by neural network.

\subsection{CCSA Technique}

In order to enhance identification ability, wavelet decomposition of the EEG signal was performed with one section every 8 seconds. We use the continual characteristic segment processing method with a threelayer ANN, and treated the wavelet coefficient as the training input of the ANN. The input layer contained 12 processing elements and the first hidden layer contained 32 processing elements. The output layer contained 3 processing elements. The weights of the connection layer were modulated to perform the tasks of training and classification. We set a learning rate parameter of 0.01 so that the system would train 10,000 times. The ANN employed a sigmoid function to restrict the output value within a range of -1 to +1 . We used the output result for comparison purposes.

\subsection{CSPA Technique}

The CSPA technique was used to perform wavelet decomposition of the EEG signal with one section every 6 seconds. We found that the use of a hidden layer with 32 neurons yielded the best identification rate. We used probability mapping as shown in formula (1) to obtain the variable material criterion and normalize the wavelet coefficient output matrix. When the variable materials were distributed consistent with parameter normality, testing and choosing $\mathrm{k}=1.96$ enabled us to map $95 \%$ of input variable values within $[-1,+1]$ to obtain three types of output condition.

$$
X_{\text {new }}=\frac{X_{\text {old }}-\mu}{k \times s}
$$

$\mu$ : average of the parameter

s: standard deviation of the parameter

Characteristic segment part processing was 
performed by modulating 0.1 of the learning rate parameters and the corresponding threshold values (AE2, NE2, SE2,) which ensured that training was carried out approximately 10,000 times. We then arrived at the least mean-square error of the actual output and the goal output. The convergence is shown in Fig. 3 [6-7].

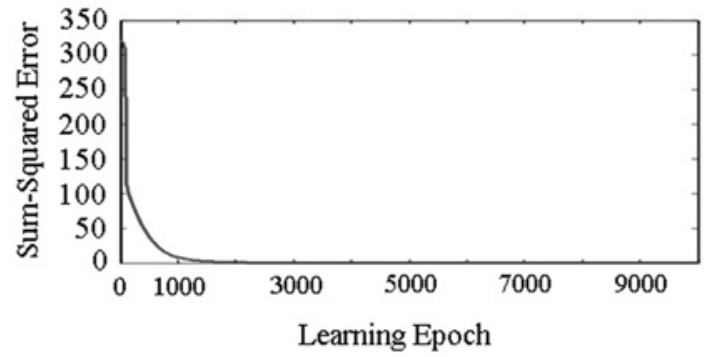

Fig. 3. The convergent situation of the EEG signal trained.

A section of the EEG signal characteristic of SAS was specially tested and analyzed via ANN. We found that the characteristic value of SAS defined $[1,0,0]$ occurred in the neighborhood of the $2430^{\text {th }}$ second (around the 2424 $4^{\text {th }}-2430^{\text {th }}$ second). The convergence of the SAS characteristic value was revealed clearly, as shown in Fig. 4. The convergence demonstrates that the output target value of SAS continuously approached the training value.

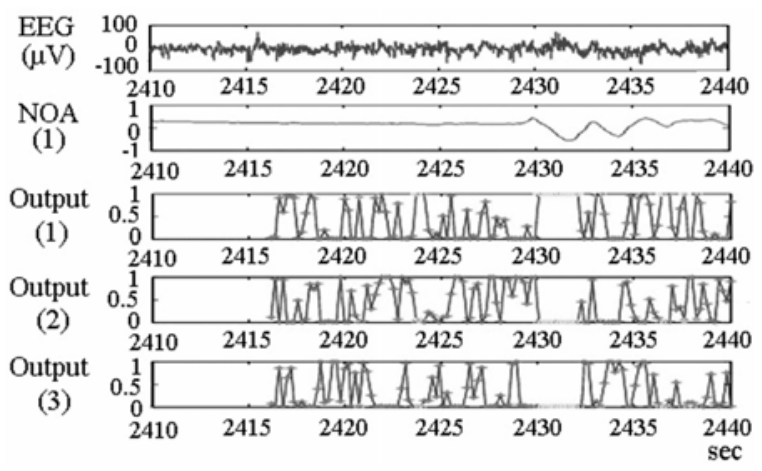

Fig. 4. The convergent result of the EEG signal trained.

\section{RESULT}

This paper presents to results of analysis of the slp59 EEG recording from the MIT-BIH sleep database using the wavelet transform and an artificial neural network method. The measuring electrode points were located at C3-O1, and the signal showed no interference from muscle artifacts. We identified SAS episodes using three different ANN methods involving characteristic part analysis, continual characteristic segment analysis, and characteristic segment part analysis. Identification results for all sleep recordings were compared with non-rapid eye movement sleep judgments made by doctors. After comparison, we finally arrived at the computer identification results shown in Table II to Table VII.

Table II. The recognition effect of the entire sleep material of CPA technique

\begin{tabular}{|l|c|c|}
\hline System Doctor & $\begin{array}{c}\text { SAS } \\
\text { ( Positive ) }\end{array}$ & $\begin{array}{c}\text { SAS } \\
\text { ( Negative ) }\end{array}$ \\
\hline SAS ( Positive ) & 94 & 164 \\
\hline SAS ( Negative ) & 46 & 154 \\
\hline & Sensitivity & Specificity \\
& $67.14 \%$ & $48.43 \%$ \\
\cline { 2 - 3 }
\end{tabular}

Table III. The recognition effect of the non-rapid eye movement sleep material of CPA technique

\begin{tabular}{|c|c|c|}
\hline Dystem & $\begin{array}{c}\text { SAS } \\
\text { ( Positive ) }\end{array}$ & $\begin{array}{c}\text { SAS } \\
\text { ( Negative })\end{array}$ \\
\hline SAS ( Positive ) & 78 & 95 \\
\hline SAS ( Negative ) & 34 & 76 \\
\hline & $\begin{array}{c}\text { Sensitivity } \\
69.64 \%\end{array}$ & $\begin{array}{c}\text { Specificity } \\
44.44 \%\end{array}$ \\
\cline { 2 - 3 }
\end{tabular}

Table IV. The recognition effect of the entire sleep material of CCSA technique

\begin{tabular}{|l|c|c|}
\hline System Doctor & $\begin{array}{c}\text { SAS } \\
\text { ( Positive ) }\end{array}$ & $\begin{array}{c}\text { SAS } \\
\text { ( Negative ) }\end{array}$ \\
\hline SAS ( Positive ) & 80 & 183 \\
\hline SAS ( Negative ) & 60 & 135 \\
\hline & Sensitivity & Specificity \\
\cline { 2 - 3 } & $57.14 \%$ & $42.45 \%$ \\
\hline
\end{tabular}

Table $\mathrm{V}$. The recognition effect of the non-rapid eye movement sleep material of CCSA technique

\begin{tabular}{|l|c|c|}
\hline System Doctor & $\begin{array}{c}\text { SAS } \\
\text { ( Positive) }\end{array}$ & $\begin{array}{c}\text { SAS } \\
\text { ( Negative ) }\end{array}$ \\
\hline SAS ( Positive ) & 70 & 92 \\
\hline SAS ( Negative ) & 42 & 79 \\
\hline & Sensitivity & Specificity \\
\cline { 2 - 3 } & $62.5 \%$ & $46.2 \%$ \\
\hline
\end{tabular}


Table VI. The recognition effect of the entire sleep material of CSPA technique

\begin{tabular}{|l|c|c|}
\hline System Doctor & $\begin{array}{c}\text { SAS } \\
\text { ( Positive ) }\end{array}$ & $\begin{array}{c}\text { SAS } \\
\text { ( Negative ) }\end{array}$ \\
\hline SAS ( Positive ) & 89 & 179 \\
\hline SAS ( Negative ) & 51 & 139 \\
\hline & Sensitivity & Specificity \\
& $63.57 \%$ & $43.71 \%$ \\
\cline { 2 - 3 }
\end{tabular}

Table VII. The recognition effect of the non-rapid eye movement sleep material of CSPA technique

\begin{tabular}{|l|c|c|}
\hline System Doctor & $\begin{array}{c}\text { SAS } \\
\text { ( Positive ) }\end{array}$ & $\begin{array}{c}\text { SAS } \\
\text { ( Negative ) }\end{array}$ \\
\hline SAS ( Positive ) & 72 & 91 \\
\hline SAS ( Negative ) & 40 & 80 \\
\hline & Sensitivity & $\begin{array}{c}\text { Specificity } \\
64.29 \%\end{array}$ \\
\cline { 2 - 3 } & \multicolumn{2}{|c}{} \\
\cline { 2 - 3 }
\end{tabular}

We finally confirmed identification results for the slp59 EEG signal recording. The results reveal that computer identification rate for non-rapid eye movement sleep is better than for all sleep data in the MIT-BIH database. We therefore confirmed that the EEG signals characteristic of SAS appear frequently during non-rapid eye movement sleep. In addition, the other five sets of EEG recordings in the MIT-BIH sleep database with the same electrode positions (C3-O1) were also analyzed using wavelet neural network training; Table VIII to Table X show the system's identification results. We finally reached the conclusion that the system recognition rate for the slp59 EEG recording is better than for the other sleep recordings.

Due to physiological factors, however, different people's characteristics are not entirely identical. If a patient's EEG signal characteristics are not clear, the ANN method will be unable to correctly identity SAS episodes, even though it can separate the characteristic frequency band scope by means of wavelet decomposition.

\section{CONCLUSIONS}

In this paper we have tried to find a new solution to the problem of identifying SAS episodes. In order to achieve this goal, the EEG signal characteristics of SAS episodes were extracted using an ANN. A wavelet neural network was used to as a SAS
Table VIII. The recognition effect of others of CPA technique

\begin{tabular}{|c|c|c|c|c|}
\hline \multirow{2}{*}{$\begin{array}{c}\text { EEG } \\
\text { Record }\end{array}$} & \multicolumn{2}{|c|}{ The entire sleep material } & \multicolumn{2}{|c|}{$\begin{array}{c}\text { The non-RNM sleep } \\
\text { material }\end{array}$} \\
\cline { 2 - 5 } & Sensitivity \% & Specificity \% & Sensitivity \% & Specificity \% \\
\hline slp03 & 52.08 & 59.23 & 54.55 & 59.62 \\
\hline slp16 & 49.79 & 54.09 & 49.48 & 51.26 \\
\hline slp45 & 50.24 & 50.45 & 48.72 & 50.82 \\
\hline slp61 & 49.55 & 58.80 & 48.17 & 59.51 \\
\hline slp67x & 50.03 & 55.68 & 51.02 & 57.58 \\
\hline
\end{tabular}

Table IX. The recognition effect of others of CCSA technique

\begin{tabular}{|c|c|c|c|c|}
\hline \multirow{2}{*}{$\begin{array}{c}\text { EEG } \\
\text { Record }\end{array}$} & \multicolumn{2}{|c|}{ The entire sleep material } & \multicolumn{2}{c|}{$\begin{array}{c}\text { Then- RNM sleep } \\
\text { material }\end{array}$} \\
\cline { 2 - 5 } & Sensitivity\% & Specificity\% & Sensitivity\% & Specificity\% \\
\hline slp03 & 58.33 & 56.70 & 50.00 & 55.60 \\
\hline slp 16 & 46.81 & 62.96 & 46.91 & 66.39 \\
\hline slp45 & 55.61 & 43.42 & 55.77 & 43.24 \\
\hline slp61 & 44.55 & 60.40 & 45.55 & 59.20 \\
\hline slp67x & 48.48 & 50.50 & 51.02 & 57.58 \\
\hline
\end{tabular}

Table X. The recognition effect of others of CSPA technique

\begin{tabular}{|c|c|c|c|c|}
\hline \multirow{2}{*}{$\begin{array}{c}\text { EEG } \\
\text { Record }\end{array}$} & \multicolumn{2}{|c|}{ The entire sleep material } & \multicolumn{2}{c|}{$\begin{array}{c}\text { The non-RNM sleep } \\
\text { material }\end{array}$} \\
\cline { 2 - 5 } & Sensitivity \% & Specificity \% & Sensitivity \% & Specificity \% \\
\hline slp03 & 31.25 & 67.26 & 45.45 & 68.50 \\
\hline slp16 & 40.43 & 60.57 & 39.69 & 67.23 \\
\hline slp45 & 52.68 & 53.51 & 52.56 & 53.89 \\
\hline slp61 & 36.82 & 64.40 & 35.08 & 66.56 \\
\hline slp67x & 37.88 & 62.50 & 40.82 & 72.73 \\
\hline
\end{tabular}

identification system. The system's identification results achieved a sensitivity of approximately $69.64 \%$ and a specificity of approximately $44.44 \%$. By reducing diagnosis time as well as SAS seizure tracking time, the system thus improves medical service efficiency.

\section{DISCUSSION}

Though the ANN SAS identification system 
developed in this study yielded promising initial results, it still requires improvement:

(1) If suffocation, muscle pains, or limb twitches, etc., occur during the sleep period, the EEG signal may awaken the patient; therefore accidental awakening must be prevented.

(2) The EEG signal characteristics of a SAS episode are certainly not entirely clear, the signal is easily contaminated by artifacts, and episodes may be difficult to recognize. A preprocessor circuit is therefore needed to eliminate EEG signal artifacts and enable the system to recognize SAS episodes.

\section{REFERENCE}

1. Takenao Sugi, Masatoshi Nakamura, Takahiro Shimokawa, Fusae Kawana: Automatic detection of EEG arousals by use of normalized parameters for different subjects. Biomedical Engineering. 2003; 146-147.

2. Dennis A, Silage: Spectral and coherence analysis applied to sleep apnea. Engineering in Medicine and Biology Magazine. 1990; 1: (9): 76-78.

3. Akin M, Arserim MA, Kiymik MK, Turkoglu I: A new approach for diagnosing epilepsy by using wavelet transform and neural networks. Proceedings of the 23rd Annual International Conference of the IEEE. 2001; 2: 25-28.

4. David NF Fairbanks, Samuel A Mickelson, and B Tucker Woodson: Snoring and Obstructive Sleep Apnea, Philadelphia: Lippincott Williams \& Wilkins. 2003: 9-15.

5. D Novak, D Cuesta-Frau, and L. Lhotska: Speech recognition methods applied to biomedical signals processing. Engineering in Medicine and Biology Society. 2004; 1: 118-121.

6. Takamasa Shimada, Tsuyoshi Shiina, and Yoichi Saito: Detection of characteristic waves of sleep EEG by neural network analysis. Biomedical Engineering. 2000; 3(47): 369-379.

7. Takenao Sugi, Masatoshi Nakamura, Takahiro Shimokawa, and Fusae Kawana: Automatic detection of EEG arousals by use of normalized parameters for different subjects. Biomedical Engineering. 2003: 146-147. 